\title{
COMPUTER VISION SYNDROME: A SHORT REVIEW.
}

Sameena Kokab, Mohd Inayatullah Khan

1. Assistant Professor Department of Ophthalmology, Navodaya Medical College, Raichur

2. Department of Physiology, Rajiv Gandhi institute of Medical Sciences. RIMS, Adilabad, Andra Pradesh,

\section{CORRESPONDING AUTHOR}

Dr. Sameena Kokab, Asst Professor, Dept of Ophthalmology,

Navodaya Medical College, Raichur.

Karnataka State.

E-mail: sameena_kokab@rediffmail.com,

Ph: 00918970644465.

ABSTRACT: Computers are probably one of the biggest scientific inventions of the modern era, and since then they have become an integral part of our life. The increased usage of computers have lead to variety of ocular symptoms which includes eye strain, tired eyes, irritation, redness, blurred vision, and diplopia, collectively referred to as Computer Vision Syndrome (CVS). CVS may have a significant impact not only on visual comfort but also occupational productivity since between $64 \%$ and $90 \%$ of computer users experience visual symptoms which may include eyestrain, headaches, ocular discomfort, dry eye, diplopia and blurred vision either at near or when looking into the distance after prolonged computer use [1].

KEY WORDS: Computer Vision Syndrome

INTRODUCTION: The generic name of "Computer vision syndrome", it is defined by the American Optometric Association as a complex of eye and vision problems related to the activities which stress the near vision and which are experienced in relation, or during, the use of the computer [2]. The symptoms of computer vision syndrome may vary depending on several factors which includes amount of time spend, viewing distance, seating posture, level of computer screen, and underlying visual acuity disturbances if any. Treatment of computer vision syndrome involves proper identification of the etiologic factors and correction of visual errors if existent. Special attention should be paid to ergonomic factors like correct posture in the chair, lighting arrangement, antiglare screen on the computer and establishing proper working habits.

ETIOLOGY OF COMPUTER VISION SYNDROME: It is difficult to point out a single etiologic factor which causes computer vision syndrome but it is a combination of several factors like prolonged working hours, inadequate rest breaks constantly staring at a single source are some of the important causes of computer vision syndrome.

LOW CONTRAST: Studies have shown that The computer monitor is populated by tiny dots called pixels, the computer screen is difficult for the eye to focus on and these pixels are not uniformly bright and produce slight difference in contrast as a result of which even at high resolutions the edge of the letter looks fuzzy this adds to strain on eyes and is one of the important cause of computer vision syndrome ${ }^{[3]}$. 
DURATION OF USE: Most people work 6-7 hour days. If you take a 1 hour lunch break, this still leaves 3.5-4 hour periods during which you are staring at a computer screen. Extended viewing of a computer screen (over 2 hours), especially at a constant depth of field, is the primary cause of CVS.

REDUCED BLINKING: It has been observed that with prolonged use of the computers the blinking rate reduces. The normal blinking rate is about one to two dozen times a minute. Since blinking is important for hydration of eyes reduced blinking is also considered one of the causes of dry eyes and Computer Vision Syndrome.

REDUCED EYE MOVEMENT: During activities in which they are not focused on a computer screen the eye moves through a lesser range of motion when concentrating on a screen. This reduction in eye movement serves to dehydrate the eye, which can, among other things, lead to CVS.

UNCORRECTED VISION PROBLEMS: Preexisting uncorrected vision problems can increase the severity of computer vision syndrome. People who are prescribed eyeglass have to tilt their heads at odd angles because their glasses aren't designed for looking at a computer. They adopt postures to look at the computers which may not be ergonomically correct. Such postures can result in muscle spasms or pain in the neck, shoulder or back.

DIAGNOSIS OF COMPUTER VISION SYNDROME: Computer Vision Syndrome can be diagnosed through a complete clinical eye examination.

PATIENT'S HISTORY: is taken to determine the underlying cause and the presence of any general health problems, medications taken, or environmental factors that may be contributing to the symptoms related to computer use.

VISUAL ACUITY MEASUREMENTS: both for near vision as well as far vision are tested, to assess the extent to which vision may be affected.

A refraction test is done to rectify the refractive errors that is existent and needs correction.

In most cases of CVS, the clinician will be able to provide the diagnosis based on the history and the clinical examination. There are numerous accommodative disorders (e.g., decreased amplitude of accommodation) and binocular vision dysfunctions (e.g., phoria, strabismus) that can clearly cause the symptoms. Improperly corrected presbyopia can also result in symptoms. Hyperopia can result in visual symptoms - especially in near workers.

\section{TREATMENT OF COMPUTER VISION SYNDROME}

EYE CARE: Patients, both young and old, may benefit from special reading glasses to enhance the clarity of their computer screens and improve their overall comfort. For patients fewer than 40 hours per week, reading glasses to help reduce the strain on their eyes can be very important. Even a mild prescription at near can cause problems for computer usage over a 40 
hour work week. Patients over the age of 40 can also benefit from a modified reading glass, typically referred to as computer glasses ${ }^{[4]}$.

ERGONOMIC FACTORS IN COMPUTER USE: Some important ergonomic consideration during computer use are the lighting conditions, chair position, location of reading materials, level on which monitor is placed and the number of breaks taken during each session.

IDEAL COMPUTER SCREEN POSITIONS: it is of considerable importance, ideally it has been noted that computer screen which are 15 to 20 degree below eye levels and which are kept at a distance of $20-28$ inches from eyes provides greatest comforts [5]. It is due to the fact that neck muscles are ideally relaxing in this posture.

CHAIR DESIGNS: the computer chairs are required to be comfortable well padded and should also provide rest to feet. The chairs should be adjustable so as to place foots flat on the ground. It should have a typical arm support while typing.

READING SOURCE: the reading materials required during computer usage should be ideally placed below the monitor and a document holder if available should be used, the goal is to position documents in such a way so as not to move your head between document reading and computer screen reading ${ }^{[5]}$.

ROOM ILLUMINATION: it is essential to position all computer screens to avoid direct glare from lighting sources. Usage of the lower wattage bulbs [5] and proper fluorescence is an important factor in preventing computer vision syndrome. Antiglare screen and filters can also effectively decrease the amount of light reflected from screen.

THE REST PERIODS: every computer user should rest eyes in between when reading computers for prolonged durations. Rests in form of looking at distant objects for at least 20 seconds, frequent blinking in between and relaxing eyes for some time does a real benefit by relaxing accommodation and preventing computer vision syndrome.

THE BLINKING REFLEX: it is one of the inborn reflexes and is considered as one of the fastest reflexes of the body. However this reflex is modified by different physiological activities. It is generally slower when concentrating on a single task. During computer works the eyes are kept wide open and constantly focus on single source and also the blinking reflex drops there is also increased evaporation of the tears due wide opening of eyes. This is one of the factors in development of computer vision syndrome. It also is likely that the higher gaze angle results in a greater percentage of incomplete blinks [6].

\section{REFERENCES:}

1. Rosenfield $M$ Computer vision syndrome: a review of ocular causes and potential treatments Ophthalmic Physiol Opt. 2011 Sep;31(5):502-15. doi: 10.1111/j.14751313.2011.00834.x. Epub 2011 Apr 12. PMID:21480937

2. Barar A, Apatachioaie ID, Apatachioaie C, Marceanu-Brasov L Oftalmologia. 2007;51(3):104-9. [Ophthalmologist and "computer vision syndrome"]. [Article in Romanian]. PMID:18064965 


\section{REVIEW ARTICLE}

3. Torrey, Jon 'Understanding computer vision syndrome' Employment Relations Today; Spring 2003; 30, 1; ProQuest Central pg. 45

4. Saari, Richard. Tribune [Welland, Ont] Computer Vision Syndrome: [Final Edition] 28 June 2004: A10.

5. American Optometric Association: http://www.aoa.org/x5253.xml. Assessed on 25-122012.

6. Anshel, Jeffrey. Computer vision syndrome: Causes and curses Managing Office Technology 42.7 (Jul 1997): 17-19. 